\title{
Day-Ahead Hourly Operation Planning of Distribution Networks with Photovoltaic Generation Integrated by Smart Inverters via Mixed-Integer Volt/Var Optimization Problems.
}

\author{
João Vitor A. de A. Barros ${ }^{1}$, Amílcar F. Q. Gonçalves ${ }^{1}$, Claudionor F. do Nascimento ${ }^{1}$ e \\ Guilherme G. Lage ${ }^{1}$ \\ ${ }^{1}$ Departamento de Engenharia Elétrica, Universidade Federal de São Carlos, São Carlos, SP, 13565-905, Brasil. \\ Data de recebimento do manuscrito: 02/01/2021 \\ Data de aceitação do manuscrito: 05/04/2021 \\ Data de publicação: 14/04/2021
}

\begin{abstract}
The penetration of distributed photovoltaic generation in distribution networks has become a reality; however, it still imposes challenges to be overcome, such as the operation of these networks with minimal deviations in voltage magnitude profiles. Thus, this work proposes the day-ahead hourly operation planning of distribution networks aiming at minimizing the deviation in voltage magnitude profiles by means of optimal tap ratio adjustments of transformers at substations, optimal settings of remote switchable capacitor banks along primary feeders, and optimal active and reactive power injections from the distributed photovoltaic generation by smart inverters. In such Volt/Var Optimization (VVO) problem, discrete control variables associated with tap ratios of transformers and equivalent susceptances of switchable capacitor banks are handled by sinusoidal penalty functions that modify the original mixed-integer nonlinear programming problem into a nonlinear programming problem with only continuous decision variables. Results obtained for distribution networks with 69 and 135 nodes show that the proposed VVO model and its resolution methodology are effective and efficient in reducing the deviation in voltage magnitude profiles whilst also reducing the distribution network overall reactive power demand.
\end{abstract}

Keywords - mixed-integer nonlinear programming, distributed photovoltaic generation, smart inverters, Volt/Var optimization.

\section{INTRODUÇÃO}

$\mathbf{O}$ uso racional de recursos econômicos, técnicos e naturais na operação dos sistemas de energia elétrica é intrínseco às boas práticas de eficiência energética. Assim, a Geração Fotovoltaica Distribuída (GFD) integrada às Redes de Distribuição (RDs) tem se tornado uma opção viável para a redução da demanda por novos empreendimentos de grande porte devido à sua viabilidade econômica, aos avanços tecnológicos na fabricação de painéis solares fotovoltaicos cada vez mais eficientes e, não menos importante, às suas características ambientais [1,2]. Entretanto, a presença massiva da GFD em RDs pode resultar em desvios nos perfis de magnitude de tensão, em variações nos fluxos de potência ativa e reativa nos alimentadores primários e secundários das RDs, e no aumento da sensibilidade da proteção [3]. Em [4,5] fica evidente que a inserção de diferentes fontes de Geração Distribuída (GD), como, por exemplo, a fotovoltaica, pode reduzir custos técnico-operacionais das RDs; porém, a alo-

Dados de contato: João Vitor Barros, Amílcar Gonçalves, Claudionor Nascimento and Guilherme Lage, joaobarros@estudante.ufscar.br, amilcarf@ufscar.br, claudionor@ufscar.br and glage@ufscar.br cação e o dimensionamento da capacidade instalada são determinantes para se atingir esse objetivo [6,7].

Nesse contexto, tornam-se essenciais a proposição e o desenvolvimento de novos modelos e metodologias de resolução para o planejamento de véspera da operação horária das RDs com GFD. A Otimização Volt/Var (OVV) se apresenta, portanto, como a melhor ferramenta para a definição do conjunto de tomadas de decisão ótimas para o planejamento de véspera da operação de RDs segundo os fundamentos e as filosofias de smart grids. Nesse problema de OVV, taps de transformadores abaixadores nas subestações, bancos de capacitores chaveados remotamente ao longo dos alimentadores primários, e as injeções de potência ativa e reativa da GFD integrada por inversores inteligentes [8,9] são passíveis de serem controlados para se otimizar um determinado desempenho técnico-operacional da RD.

No entanto, os taps dos transformadores nas subestações e as susceptâncias equivalentes dos bancos de capacitores são variáveis de controle discretas [10], e, portanto, esse problema de OVV é formulado matematicamente como um problema de Programação Não Linear Inteira Mista (PNLIM) de grande porte, estático e não convexo [11]. Segundo [12], pode-se dividir as técnicas de resolução de problemas de PNLIM em quatro categorias: Branch-and-Bound, Branchand-Cut, Outer Approximations e Decomposição de Ben- 
ders generalizada. Entretanto, essas técnicas demandam alto custo computacional para problemas de grande porte em que o número de variáveis de controle discretas é elevado $[11,13,14]$.

Para se resolver problemas de OVV e, assim, garantir a robustez dessa ferramenta, existem metodologias propostas na literatura correlata que não utilizam técnicas de PNLIM e propõem alternativas como técnicas de arredondamento [11], de excursão de pontos interiores à região factível delimitada pelos planos de corte [15], funções penalidade quadrática com suavização da função barreira logarítmica [16], análise de sensibilidade por variáveis duais [17], métodos de otimização global com lógica fuzzy [18], algoritmos evolutivos [19] e funções penalidade senoidal quadrática $[13,14]$

Por outro lado, a integração da GFD é fundamental para operação das RDs, e seu comportamento deve ser incluído no modelo de OVV apresentado neste trabalho. Em [20] foi proposta a injeção de potência reativa por inversores inteligentes para fatores de potência não unitários, com o objetivo de minimizar o desvio nos perfis de magnitudes tensão; no entanto foram analisados resultados considerando-se a integração da GFD em um único nó. Em [21] foi apresentada a integração da GFD pela injeção de potência reativa por inversores inteligentes com fator de potência constante, também buscando minimizar os desvios de magnitude tensão. Em [22] foi apresentado um modelo para minimizar os desvios de magnitudes de tensão pela injeção de potência reativa por inversores inteligentes com fator de potência não constante, entretanto, foi utilizado uma otimização multi-objetiva com o fluxo de carga linearizado considerando apenas o ajuste nos taps dos transformadores com comutação sob carga.

Nesse contexto, este trabalho tem como objetivo a modelagem e a resolução de um problema de OVV que consiste na minimização dos desvios de magnitude de tensão em RDs para o planejamento de véspera da operação horária com GFD integrada por inversores inteligentes para um fator de potência unitário. Cabe ressaltar que, por se tratar de um planejamento no curto prazo, a injeção de potência na RD pela GFD pode ser considerada determinística e a modelagem dos inversores inteligentes é feita de acordo com [20]. A metodologia de tratamento das variáveis de controle discretas do problema consiste no emprego da técnica de Programação Não Linear (PNL) com funções penalidade senoidal quadrática apresentada em $[13,14]$.

As principais contribuições deste trabalho consistem, portanto, na proposição de um modelo atual e realista para o planejamento de véspera da operação horária das RDs com GFD integrada por inversores inteligentes e na sua resolução por uma metodologia robusta e eficiente que permite o emprego de solvers comerciais de problemas de PNLs de grande porte.

Um cenário foi criado para realização do estudo. As redes de distribuição são consideradas trifásicas equilibradas; a geração Fotovoltaica (FV) obedece um perfil de geração parabólico sem variações pontuais; os bancos de capacitores estão dispostos ao longo na rede com chaveamentos remoto; e a injeção de potência reativa pelo inversor inteligente pode ser tanto positiva (injetando potência reativa) quanto negativa (absorvendo potência reativa) e somente pode ocorrer no período que haja potência gerada disponível.
Este trabalho está organizado da seguinte forma. $\mathrm{Na}$ Seção II são apresentadas as considerações que consolida a integração dos inversores inteligentes com a rede de distribuição através da OVV. Na Seção III é apresentada a modelagem do problema de OVV para o planejamento de véspera da operação horária das RDs com GFD integrada por inversores inteligentes. Na Seção IV é apresentada a metodologia para o tratamento das variáveis de controle discretas do OVV. Na Seção V são apresentados as considerações, os resultados numéricos e as análises para RDs de 69 e 135 nós. Por fim, na Seção VI são destacadas as principais contribuições e conclusões acerca do modelo e da metodologia de resolução do problema de OVV apresentado neste trabalho.

\section{INVERSORES INTELIGENTES}

O conceito de redes inteligentes trabalha com a possibilidade de comunicação entre os dispositivos presentes no sistema elétrico. Há diversos trabalhos na literatura que propõem arquiteturas de comunicação para inversores inteligentes, sendo alguns voltados ao controle de magnitudes de tensão, como em [23], no qual uma infraestrutura de comunicação para o controle da magnitude da tensão em sistemas de transmissão de potência é proposta; em [24] é proposta uma arquitetura de controle de tensão descentralizada e não hierárquica baseada em agentes fuzzy cooperativos; e em [25] um estudo foi realizado para para avaliar a performance da comunicação no controle de inversores inteligentes com o objetivo de controlar as magnitudes de tensão em redes de distribuição.

Um inversor inteligente para sistemas fotovoltaicos é um conversor de Corrente Contínua (CC) para Corrente Alternada (CA), conectado na rede de distribuição, dotado de um sistema de comunicação e que possa operar em diferentes modos de injeção de potência ativa e/ou reativa, conforme seja estabelecido pelo centro de operações da rede. Para isso, é essencial que a GFD tenha a capacidade de injetar energia na rede por meio dos painéis fotovoltaicos, bem como armazenar energia em baterias ou supercapacitores, com a finalidade de gerenciar as injeções de potência ativa e reativa nos quatro quadrantes (vide Figura 1 da referência [20]). Dessa forma, seguindo os padrões da norma IEEE 15472018, o controle empregado aqui na GFD permite a operação no modo OVV por meio do ajuste da magnitude e fase de tensão nos inversores inteligentes, controlando, portanto, as potências ativa e reativa, injetada ou absorvida da rede.

O sincronismo de fase, para que ocorra a integração dos inversores inteligentes à rede, é realizado por meio do algoritmo de bloqueio de fase (Phase Locked Loop - PLL) que produz uma referência sincronizada em fase e frequência com o sinal de entrada (tensão da rede). O PLL tem papel fundamental no rastreamento do ângulo de tensão do sistema CA no ponto de acoplamento comum.

Para controlar as potências ativa e reativa que serão fornecidas pelo inversor inteligente é utilizada a transformação de Park ( $d q O)$. Na transformada de Park os sinais no referencial trifásico $(a b c)$ são convertidos para um referencial de duas coordenadas síncronas $(d q 0)$. O controle em coordenadas $d q 0$ permite que os sinais passem de um sistema de característica de sinal alternado para um sistema com car- 
acterística de sinal contínuo, separando o controle de potência ativa no eixo direto (d) e o controle de potência reativa no eixo de quadratura (q) [26]. Além disso, o fato dos sinais terem a característica CC permite que o controle em $d q 0$ utilize controladores proporcional-integral (PI), zerando o erro de regime permanente. O leitor interessado em se aprofundar na modelagem do inversor inteligente veja a referência [26].

\section{Modelagem do Problema de OVV}

Dado que problemas de OVV são formulados como problemas de PNLIM de grande porte, estáticos e não convexos [11], tais problemas podem ser expressos matematicamente pela seguinte formulação genérica:

$$
\begin{aligned}
& \min Z=\sum_{t=1}^{T} f\left(x^{(t)}, y^{(t)}\right) \\
& \text { s.a: } \quad g\left(x^{(t)}, y^{(t)}\right)=0, \\
& \forall t \in \mathscr{T} \\
& h\left(x^{(t)}, y^{(t)}\right) \leq 0, \\
& \forall t \in \mathscr{T} \\
& x_{\min } \leq x^{(t)} \leq x_{\max }, \\
& \forall t \in \mathscr{T} \\
& y^{(t)} \in \mathscr{D}_{y}, \\
& \forall t \in \mathscr{T}
\end{aligned}
$$

em que $t$ representa cada intervalo de discretização do horizonte de planejamento $\mathscr{T}=\{1, \ldots, T\} ; x^{(t)} \in \mathbb{R}^{m}$ representa o vetor das variáveis de decisão contínuas do problema, cujos limites inferiores e superiores são dados, respectivamente, pelos vetores $x_{\text {min }} \in \mathbb{R}^{m}$ e $x_{\text {max }} \in \mathbb{R}^{m}, y^{(t)} \in \mathbb{R}^{n}$ representa o vetor das variáveis de decisão discretas do problema, cujos valores são definidos pelo conjunto $\mathscr{D}_{y}$; A função objetivo $Z$ : $\mathbb{R}^{m} \cup \mathbb{R}^{n} \mapsto \mathbb{R}$ consiste no somatório dos desvios quadráticos das magnitudes de tensão nodais da RD; $g: \mathbb{R}^{m} \cup \mathbb{R}^{n} \mapsto \mathbb{R}^{r}$ representa as restrições de igualdade do problema e consiste nas equações de balanço de potência ativa e reativa nos nós da $\mathrm{RD}$, com $r<m+n$; e, por fim, $h: \mathbb{R}^{m} \cup \mathbb{R}^{n} \mapsto \mathbb{R}^{s}$ representa as restrições de desigualdade funcionais do problema e consiste na modelagem das injeções de potência ativa e reativa dos inversores inteligentes que integram a GFD à RD.

A modelagem do problema de planejamento de véspera da operação horária de RDs com GFD integrada por inversores inteligentes é detalhada nas próximas subseções.

\section{a. Função objetivo}

A função objetivo considerada neste trabalho consiste no somatório dos desvios quadráticos das magnitudes de tensão nodais da RD para todos os intervalos de discretização do horizonte de planejamento, e pode ser matematicamente expressa por:

$$
Z=\sum_{t=1}^{T} \sum_{k \in \mathscr{C}}\left(1-V_{k}^{(t)}\right)^{2}
$$

em que $V_{k}^{(t)}$ é a magnitude de tensão no nó $k$ no intervalo de discretização $t$ do horizonte de planejamento $\mathscr{T}$; e $\mathscr{C}$ é o conjunto dos nós de carga da RD.

\section{b. Restrições de igualdade}

As restrições de igualdade consistem nas equações de balanço de potência ativa e reativa nos nós da RD, e podem ser matematicamente expressas por:

$$
P_{k}^{(t)}+P_{k}^{f v(t)}-\sum_{m \in \Omega_{k}} P_{k m}^{(t)}\left(V^{(t)}, \theta^{(t)}, t_{k m}^{(t)}\right)=0
$$

$$
\forall k \in \mathscr{C}, \forall t \in \mathscr{T}
$$

$$
\begin{aligned}
Q_{k}^{(t)}+Q_{k}^{f v(t)}+b_{k}^{s h(t)} V_{k}^{(t)^{2}}-\sum_{m \in \Omega_{k}} Q_{k m}^{(t)}\left(V^{(t)}, \theta^{(t)}, t_{k m}^{(t)}\right) & =0, \\
\forall k & \in \mathscr{C}, \forall t \in \mathscr{T}
\end{aligned}
$$

$$
S_{k}^{f v(t)}=\sqrt{P_{k}^{f v(t)^{2}}+Q_{k}^{f v(t)^{2}}}, \quad \forall k \in \mathscr{F}, \forall t \in \mathscr{T}
$$

em que $V^{(t)}$ e $\theta^{(t)}$ são variáveis contínuas associadas às magnitudes e aos ângulos de fase das tensões nodais; $P^{f v(t)}$ e $Q^{f v(t)}$ são variáveis contínuas associadas às injeções de potência ativa e reativa por inversores inteligentes que integram a GFD à RD (os nós que não possuem GFD integrada por inversores inteligentes têm $P^{f v(t)}$ e $Q^{f v(t)}$ iguais a zero); $t^{(t)}$ e $b^{s h(t)}$ são variáveis de controle discretas associadas, respectivamente, aos taps de transformadores abaixadores nas subestações e às susceptâncias equivalentes dos bancos de capacitores chaveados remotamente ao longo dos alimentadores primários; $P^{(t)}$ e $Q^{(t)}$ são as injeções líquidas de potência ativa e reativa nos nós da RD; $S^{f v(t)}$ é um parâmetro determinístico associado à injeção de potência na RD pela GFD não superior à potência nominal do inversor inteligente; e $P_{k m}^{(t)}$ e $Q_{k m}^{(t)}$ são os fluxos de potência ativa e reativa nas linhas entre os nós $k$ e $m$.

As restrições (3a) e (3b) são as equações tradicionais que modelam os fluxos de potência ativa e reativa nos nós da RD. As restrições (3c) modela a injeção de potência ativa e reativa na RD pela integração da GFD por inversores inteligentes. Por fim, $\mathscr{F}$ é o conjunto dos nós da RD com GFD integrada por inversores inteligentes; $\Omega_{k}$ é o conjunto de todos os nós conectados ao nó $k$.

\section{c. Limites operacionais e valores discretos de variá- veis de controle}

As restrições de desigualdade consistem, respectivamente, na restrição de funcional de desigualdade (3c) que modela a injeção de potência ativa e reativa da GFD por inversores inteligentes de acordo com [20], e as restrições (4c), (4d) e (4e) são associadas aos limites técnico-operacionais da RD:

$$
\begin{array}{ll}
0 \leq P_{f}^{(t)} \leq S_{f}^{(t)}, & \forall f \in \mathscr{F}, \forall t \in \mathscr{T} \\
\left|Q_{f}^{(t)}\right| \leq S_{f}^{(t)}, & \forall f \in \mathscr{F}, \forall t \in \mathscr{T} \\
V_{k, \text { min }} \leq V_{k}^{(t)} \leq V_{k, \text { max }}, & \forall k \in \mathscr{C}, \forall t \in \mathscr{T} \\
t_{k m}^{(t)} \in \mathscr{D}_{t}, & \forall\{k, m\} \in \mathscr{J}, \forall t \in \mathscr{T} \\
b_{k}^{s h^{(t)}} \in \mathscr{D}_{b^{s h}}, & \forall k \in \mathscr{B}^{s h}, \forall t \in \mathscr{T}
\end{array}
$$

em que $\mathscr{J}$ é o conjunto dos ramos associados a transformadores abaixadores nas subestações; e $\mathscr{B}^{s h}$ é o conjunto 
dos nós com bancos de capacitores chaveados remotamente ao longo dos alimentadores primários.

\section{Tratamento das Variáveis de Con- TROLE DISCRETAS}

Em $[13,14]$, uma função penalidade senoidal quadrática foi incorporada à função objetivo de um problema de despacho ótimo de reativos com objetivo de impedir que as variáveis discretas assumissem valores não discretos. Essa função penalidade senoidal quadrática $\phi: \mathbb{R} \mapsto[0,1]$ assume valor zero quando $y_{k}^{(t)}$ assume um valor discreto e pode ser expressa matematicamente por:

$$
\phi\left(y_{k}^{(t)}\right)=\operatorname{sen}^{2}\left[\left(\frac{y_{k}^{(t)}}{s_{k}^{(t) u}-s_{k}^{(t) l}}\right) \pi+\alpha_{k}\right]
$$

em que $s_{k}^{(t) u}$ é o valor discreto imediatamente superior a $y_{k}^{(t)}$; $s_{k}^{(t) l}$ é o valor discreto imediatamente inferior a $y_{k}^{(t)}$; e $\alpha_{k}$ é uma constante definida no intervalo $[0, \pi)$ tal que a função penalidade senoidal quadrática se anule para valores de $y^{(t)}$ definidos em $\mathscr{D}_{y}$. A constante $\alpha_{k}$ é definida por:

$$
\alpha_{k}=\left(\left\lceil\frac{s_{k}^{(t) u}}{s_{k}^{(t) u}-s_{k}^{(t) l}}\right\rceil-\left(\frac{s_{k}^{(t) l}}{s_{k}^{(t) u}-s_{k}^{(t) l}}\right)\right) \pi
$$

em que $\lceil\cdot\rceil$ é a função ceil que arredonda qualquer número real para o inteiro superior imediato.

Dessa forma, problemas de OVV formulados como problemas de PNLIM podem ser transformados em problemas de PNL equivalentes da seguinte forma:

$$
\begin{aligned}
& \min Z+\gamma \sum_{t=1}^{T} \sum_{k=1}^{n} \phi\left(y_{k}^{(t)}\right) \\
& \text { s.a: } \quad g\left(x^{(t)}, y^{(t)}\right)=0, \quad \forall t \in \mathscr{T} \\
& h\left(x^{(t)}, y^{(t)}\right) \leq 0, \quad \forall t \in \mathscr{T} \\
& x_{\text {min }} \leq x^{(t)} \leq x_{\text {max }}, \quad \forall t \in \mathscr{T} \\
& y_{\text {min }} \leq y^{(t)} \leq y_{\text {max }}, \quad \forall t \in \mathscr{T}
\end{aligned}
$$

em que $\gamma$ é um parâmetro maior que zero associado à amplitude da função penalidade senoidal quadrática; $y_{\min }$ é igual a $\min \left(\mathscr{D}_{y}\right)$ e $y_{\text {max }}$ é igual a $\max \left(\mathscr{D}_{y}\right)$.

$\mathrm{O}$ algoritmo para a resolução de problemas de PNLIM transformados em problemas de PNL equivalentes apresentado neste trabalho foi adaptado de $[13,14]$. A cada iteração, o valor $\gamma$ é incrementado pelo fator $c$, fixo no intervalo $(1,10]$, para que a diferença entre o valor calculado e o discreto seja menor ou igual a uma tolerância $\xi$. O algoritmo utilizado neste trabalho é apresentado em Algoritmo 1.

\section{RESUltados NumÉricos E Discussões}

Os testes aqui apresentados foram realizados para a RD de 69 nós adaptada de [27], com tensão base $V_{\text {base }}=12,66 \mathrm{kV}$, e a RD de 135 nós adaptada de [28], com tensão

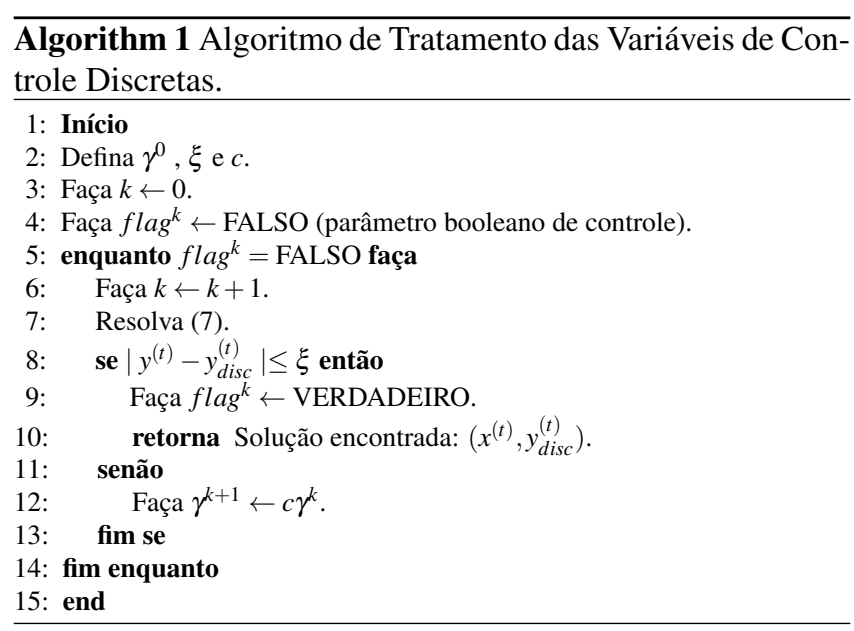

base de $V_{\text {base }}=13,80 \mathrm{kV}$, ambas com potência base $S_{\text {base }}=1000 \mathrm{kVA}$. Os perfis de carga das classes de consumidores ao longo do horizonte de planejamento considerados para os nós das RDs foram baseados em [10], de acordo com a Figura 1 e a Tabela 1 . Um ramo $0-1$ foi adicionado a cada RD para modelar a atuação de transformadores abaixadores na subestação, os quais possuem taps com limites de 0,90 e 1,10 por unidade (p.u.), com passos de discretização de 0,00625 p.u. Os limites das magnitudes de tensão são 0,95 e 1,05 p.u., e a tensão no nó 0 (enrolamento primário do transformador abaixador na subestação) é fixa e igual a 1,00 p.u. com fase 0 rad.

Os testes foram realizados em um notebook ACER E5574-78LR, com drive SSD de $512 \mathrm{~GB}$, processador Intel Core i7-6500 com 3.1 GHz e Memória Ram de 8 GB.

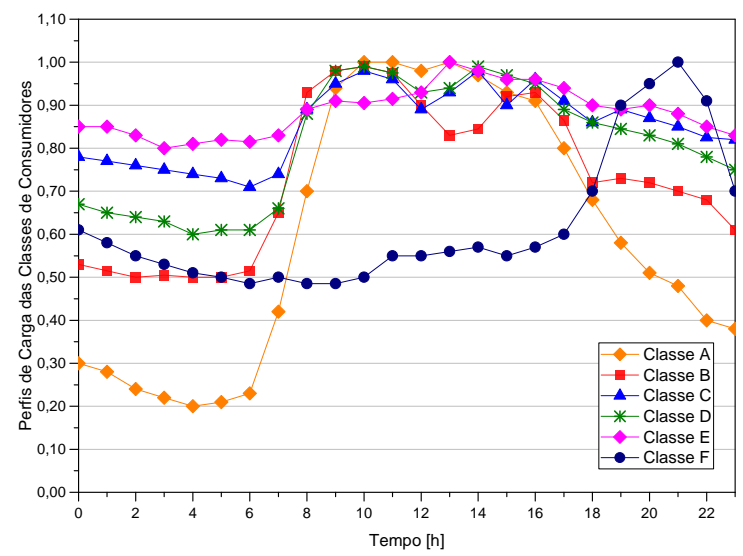

Figura 1: Perfis de carga das classes de consumidores ao longo do horizonte de planejamento [10].

De acordo com [29], o estudo de impactos da GFD nas RDs pode considerar uma penetração fotovoltaica equivalente a aproximadamente $30 \%$ da carga da RD. Dessa forma, os nós com conexão de GFD na RD de 69 nós são: 7, 16, 21, $26,33,39,48,49,51,53$, e 62 , cujos inversores inteligentes possuem uma capacidade nominal de 0,1 p.u.; e os nós com conexão de GFD na RD de 135 nós são: 7 , 18, 24, 28, 30, $37,42,53,58,67,74,85,90,95,106,110,119,126,128$, e 134, cujos inversores inteligentes possuem uma capacidade nominal de 0,5 p.u.. Os perfis de injeção de potência por cada conjunto de geração fotovoltaica para ambas as RDs são apresentados nas Figuras 2 e 3. 
ASSES DE CONSUMIDORES NAS RDS [10]

\begin{tabular}{c|l|l}
\hline Classe & RD de 69 nós & RD de 135 nós \\
\hline $\mathrm{A}$ & $59,60,62,63,64,66$, & $3,5,6,9,15,37,47,61,64$, \\
& $68,69$. & $65,76,80,86,92,96,102$, \\
& & $108,113,120,125,127,132$, \\
& & 134. \\
$\mathrm{~B}$ & $42,43,44,48,50,51$, & $4,7,17,24,34,40,44,46,53$, \\
& $53,54,55,56$. & $56,72,73,77,82,90,95,98$, \\
$\mathrm{C}$ & $37,38,39$. & $99,101,114,118,121,126$. \\
& & $2,16,21,23,30,32,39,42$, \\
& & $52,58,60,67,83,104,106$, \\
$\mathrm{D}$ & $40,41,57,58$. & $49,54,62,63,68,70,74,81$, \\
& & $85,88,105,116,123,124$. \\
$\mathrm{E}$ & $28,29,33,34,35$. & $8,14,18,38,41,48,59,71$, \\
& & $78,94,117,128,133,135$. \\
$\mathrm{F}$ & $6,7,8,9,10,11,12,13$, & $10,20,26,28,31,35,51,66$, \\
& $14,15,16,17,18,20$, & $75,89,97,100,112,122$. \\
& $21,22,24,26,27$. & \\
\hline
\end{tabular}

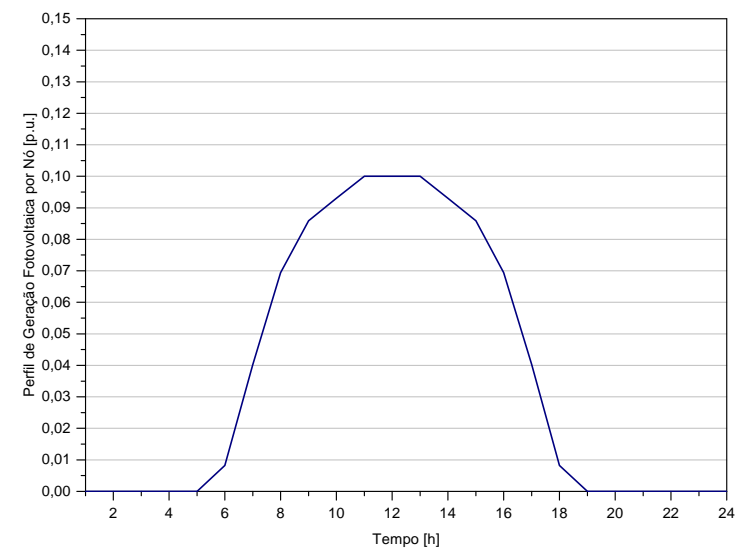

Figura 2: Perfil de injeção de potência $S^{f v(t)}$ por cada conjunto de geração fotovoltaica para a RD de 69 nós.

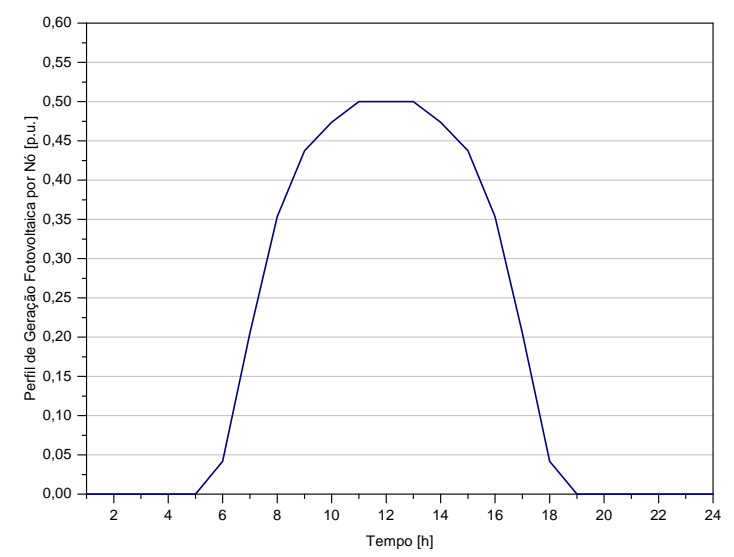

Figura 3: Perfil de injeção de potência $S^{f v(t)}$ por cada conjunto de geração fotovoltaica para a RD de 135 nós.

Por fim, para ambas RDs, os bancos de capacitores chaveados remotamente foram alocados e dimensionados de acordo com [10], os quais são formados, por sua vez, por conjuntos de 0,3 p.u. cada.

Foram realizadas três estudos de caso para a análise dos impactos da GFD integrada às RDs por inversores inteligentes: no estudo de caso 1 considerou-se a RD sem GFD (ou seja, desconsiderou-se a restrição (3c) do problema de OVV, com $P^{f v(t)}$ e $Q^{f v(t)}$ fixos e iguais a 0 p.u. para todos os intervalos de discretização $t$ do horizonte de planejamento); no estudo de caso 2 considerou-se apenas a injeção de potência ativa pela GFD (ou seja, desconsiderou-se a restrição (3c) do problema de OVV, com $P^{f v(t)}=S^{f v(t)}$ e $Q^{f v(t)}$ fixo e igual a 0 p.u. para todos os intervalos de discretização $t$ do horizonte de planejamento); e no estudo de caso 3 considerou-se o modelo completo proposto neste trabalho para o problema de OVV, no qual a integração da GFD é feita por inversores inteligentes que injetam potência ativa e reativa na RD.

Esses problemas de OVV e o algoritmo de tratamento das variáveis de controle discretas apresentado no Algoritmo 1 foram implementados no ambiente de modelagem de problemas de programação matemática AMPL $[30,31]$ e resolvidos pelo solver CONOPT [32], versão 3.17A, que utiliza o método gradiente reduzido generalizado para resolver problemas de PNL.

Os parâmetros do Algoritmo 1, $\gamma$ e $c$, determinam a amplitude da função senoidal durante as iterações do algoritmo, se o valor de $c$ for muito alto, necessita de um menor número de iterações, entretanto, pode impedir um melhor ponto de operação da RD; se o valor de $c$ for muito baixo aumenta o número de iterações necessárias e o custo computacional mas pode proporcionar um ponto de operação melhor. Os valores de $\gamma^{0}=0,00001, c=2,8$ foi adotado empiricamente. $\mathrm{O}$ erro das variáveis $\xi=0,0002$ é determinado em função do passo de ajuste das variáveis de controle.

\section{a. Resultados Numéricos para a RD de 69 Nós}

Embora a RD de 69 nós seja de pequeno porte quando comparada às RDs reais, os resultados numéricos apresentados a seguir nesta subseção serviram de base para a validação do modelo de problema de OVV proposto neste trabalho, bem como do algoritmo de tratamento das variáveis de controle discretas. O tempo de processamento do caso 1 é de 6,65625 segundos, do caso 2 é de 7,29688 segundos e do caso 3 é de 145,01628 segundos. A Tabela 2 apresenta a comparação de indicadores do desempenho operacional da RD de 69 nós obtidos para os três estudos de caso considerados neste trabalho.

TABELA 2: INDICADORES OPERACIONAIS PARA A COMPARAÇÃO DOS RESUlTADOS ENTRE OS TRÊS ESTUdOS DE Caso Simulados Para a RD de 69 Nós

\begin{tabular}{c|ccc}
\hline Indicador & Caso 1 & Caso 2 & Caso 3 \\
\hline$V_{\text {med. }}[\mathrm{p} . u]$. & 0,9998 & 1,0002 & 1,0000 \\
$V_{\text {min. }}[\mathrm{p} . \mathrm{u}]$. & 0,9643 & 0,9717 & 0,9728 \\
$V_{\text {max. }}[\mathrm{p} . \mathrm{u}]$. & 1,0105 & 1,0115 & 1,0115 \\
$P_{\text {mss. }}^{\text {loss }}[\mathrm{kW}]$ & 205,988 & 175,158 & 203,27 \\
$P_{0, \text { med. }}[\mathrm{kW}]$ & $2.992,41$ & $2.961,58$ & $2.989,67$ \\
$Q_{0, \text { med. }}[\mathrm{kVAr}]$ & $-3.217,07$ & $-3.326,72$ & $-3.533,76$ \\
\hline
\end{tabular}

A partir desses indicadores, pode-se perceber que o estudo de caso 3 , no qual a GFD integrada à RD por inversores inteligentes é modelada e incorporada ao problema de OVV, resulta em valores médios para as magnitudes de tensão iguais a 1 p.u., com uma menor diferença entre as magnitudes de tensão máxima e mínima, evidenciando a eficácia do modelo em minimizar o desvio de magnitudes de tensão. Além disso, apesar de as perdas ativas médias na RD se 
aproximarem das do estudo de caso 1, no qual a GFD não é considerada, a demanda de potência reativa da RD é menor, o que proporciona maiores margens de carregamento para o sistema elétrico de potência como um todo. Nesses estudos de caso, em específico, a RD de 69 nós está "injetando" potência reativa no sistema de transmissão.

Os perfis das magnitudes de tensão nodal são apresentados para os três estudos de caso nas Figuras 4, 5 e 6.

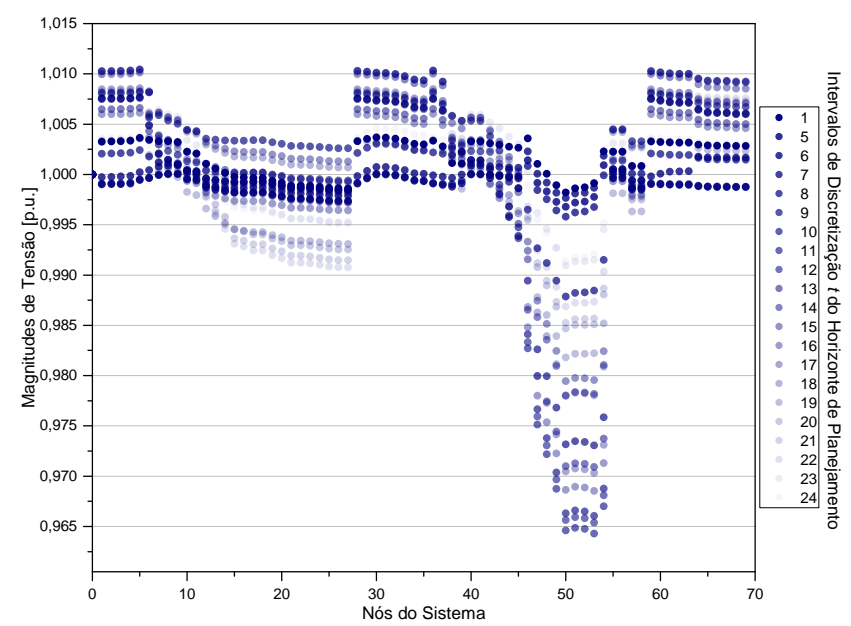

Figura 4: Magnitudes de tensão da RD de 69 nós para o estudo de caso 1 .

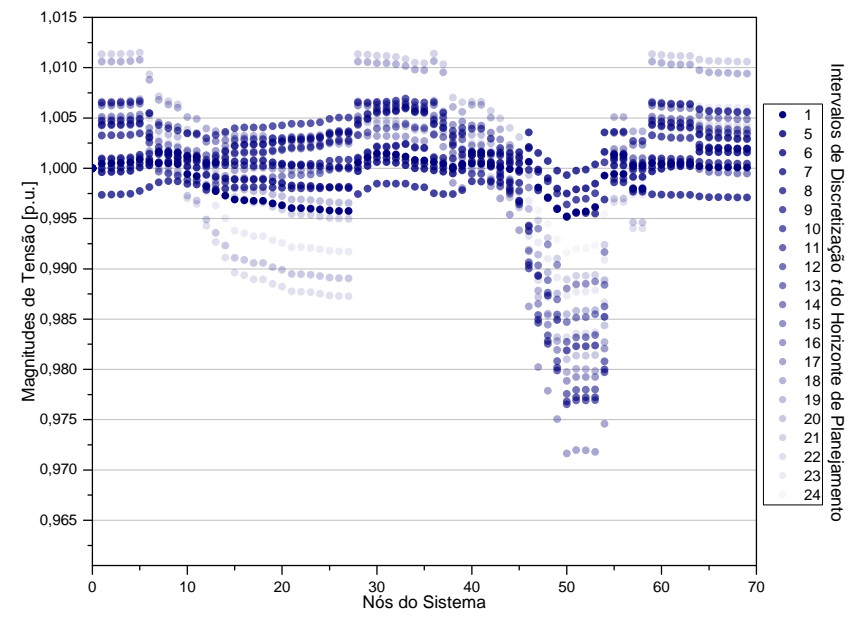

Figura 5: Magnitudes de tensão da RD de 69 nós para o estudo de caso 2 .

A RD de 69 nós possui a característica de menores valores para as magnitudes de tensão nos nós 48 a 54 devidos aos respectivos perfis de carga das cargas de consumidores e, principalmente, à topologia da RD. Comparando os valores das magnitudes de tensão nesses nós, observam-se valores de magnitude de tensão com menor desvios devido à penetração da GFD na RD.

Nos três estudos de caso o algoritmo de tratamento das variáveis de controle discretas convergiu em 12 iterações; o processo de convergência de algumas dessas variáveis para o estudo de caso 3 é apresentado na Tabela 3.

$\mathrm{O}$ algoritmo toma como ponto inicial o estado do sistema (magnitude de tensão na barra $V \theta$, posição do tap do transformador na subestação e susceptâncias equivalentes

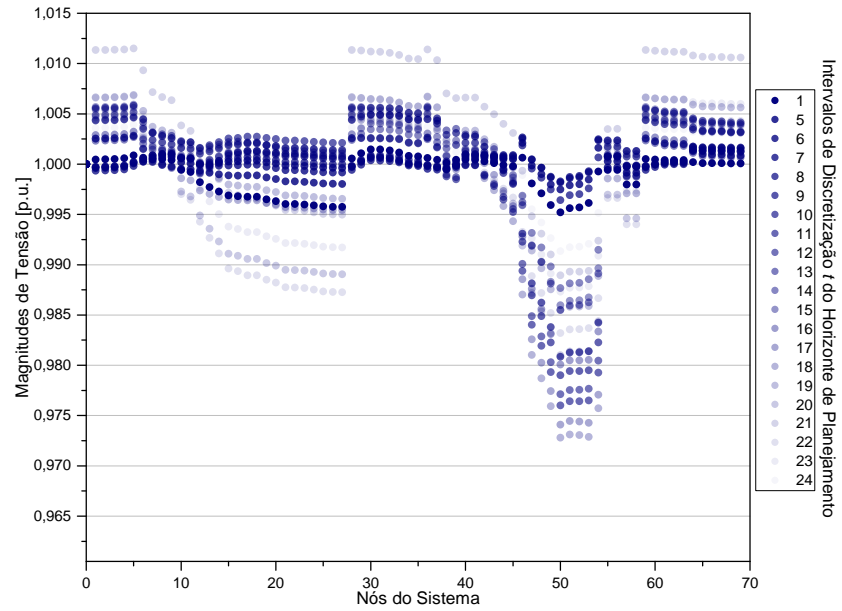

Figura 6: Magnitudes de tensão da RD de 69 nós para o estudo de caso 3.

dos bancos de capacitores chaveados remotamente), baseado nisso escolhe-se empiricamente os valores de $\gamma^{0}$ e $c$, dado que a função senoidal insere não convexidades na função objetivo. Dessa forma, a escolha desses parâmetros buscou a convergência para os menores valores da função objetivo, como apresentado na Tabela 2. Cabe ressaltar que, o ajuste ótimo das susceptâncias equivalentes shunt dos bancos de capacitores chaveados remotamente é sensível ao estado inicial do sistema.

A Figura 7 apresenta os valores absolutos das funções objetivo da RD de 69 nós dos 3 casos estudados comparando o problema com relaxação contínua das variáveis de controle e o problema discretizado. A maior diferença entre os casos avaliados é o período das 20 horas do caso 3 que é de 0,00079, ainda assim, as curvas de cada caso estão próximas evidenciando que a função penalidade senoidal é eficaz em discretizar as variáveis de controle.

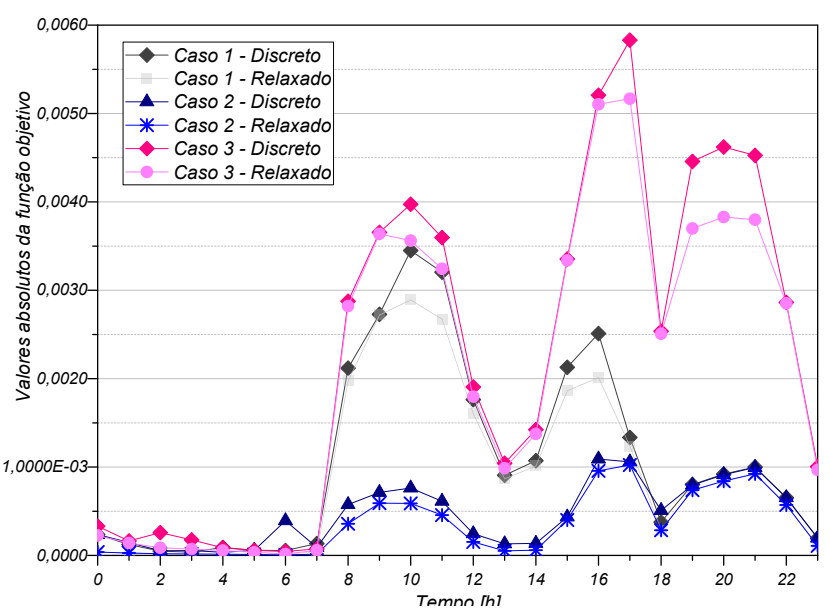

Figura 7: Valores da função objetivo dos casos avaliados do problema discreto e do problema com relaxação contínua das variáveis de controle da RD de 69 nós.

\section{b. Resultados Numéricos para a RD de 135 Nós}

Os resultados numéricos apresentados a seguir nesta subseção serviram de base para testes de robustez do modelo de problema de OVV proposto neste trabalho, devido ao seu 
Tabela 3: Convergência do Algoritmo de Tratamento das Variáveis de Controle Discretas para a RD de 69 Nós NO EsTUdO DE CASO 3

\begin{tabular}{c|ccccccccccc}
\hline Iteração & $\gamma$ & $t_{0-1}^{(6)}$ & $t_{0-1}^{(9)}$ & $t_{0-1}^{(17)}$ & $t_{0-1}^{(18)}$ & $t_{0-1}^{(21)}$ & $b_{8}^{\text {sh(1) }}$ & $b_{8}^{\text {sh(3) }}$ & $b_{36}^{\text {sh(7) }}$ & $b_{56}^{\text {shh(6) }}$ & $b_{64}^{\text {sh(20) }}$ \\
\hline 0 & 0,0000100 & 1,05000 & 1,03068 & 1,01759 & 1,01588 & 1,01558 & 0,600000 & 0,600000 & 0,897987 & 0,600000 & 0,000000 \\
1 & 0,0000280 & 1,05000 & 1,03112 & 1,01777 & 1,01589 & 1,01557 & 0,600000 & 0,596242 & 0,897570 & 0,600000 & 0,000000 \\
2 & 0,0000784 & 1,05000 & 1,03119 & 1,01791 & 1,01595 & 1,01556 & 0,600000 & 0,594056 & 0,897719 & 0,599690 & 0,000000 \\
3 & 0,0002195 & 1,05000 & 1,03125 & 1,01818 & 1,01642 & 1,01542 & 0,600000 & 0,592885 & 0,898066 & 0,599260 & 0,000000 \\
4 & 0,0006147 & 1,05000 & 1,03128 & 1,01842 & 1,01789 & 1,01349 & 0,600000 & 0,593614 & 0,898830 & 0,598648 & 0,015528 \\
5 & 0,0017210 & 1,05000 & 1,03126 & 1,01859 & 1,01834 & 1,01294 & 0,600000 & 0,596345 & 0,899537 & 0,599433 & 0,009327 \\
6 & 0,0048189 & 1,05000 & 1,03126 & 1,01869 & 1,01859 & 1,01267 & 0,600000 & 0,596727 & 0,899579 & 0,599480 & 0,008231 \\
7 & 0,0134929 & 1,05000 & 1,03125 & 1,01873 & 1,01869 & 1,01257 & 0,600000 & 0,596922 & 0,899603 & 0,599510 & 0,007727 \\
8 & 0,0377802 & 1,05000 & 1,03125 & 1,01874 & 1,01873 & 1,01253 & 0,600000 & 0,599735 & 0,899965 & 0,599954 & 0,000560 \\
9 & 0,1057850 & 1,05000 & 1,03125 & 1,01875 & 1,01874 & 1,01251 & 0,600000 & 0,599827 & 0,899980 & 0,599974 & 0,000327 \\
10 & 0,2961970 & 1,05000 & 1,03125 & 1,01875 & 1,01875 & 1,01250 & 0,600000 & 0,599896 & 0,899988 & 0,599985 & 0,000195 \\
11 & 0,8293510 & 1,05000 & 1,03125 & 1,01875 & 1,01875 & 1,01251 & 0,600000 & 0,599978 & 0,899997 & 0,599997 & 0,000043 \\
12 & 2,3221800 & 1,05000 & 1,03125 & 1,01875 & 1,01875 & 1,01250 & 0,600000 & 0,600000 & 0,900000 & 0,600000 & 0,000000 \\
\hline
\end{tabular}

maior porte e, consequentemente, maior número de variáveis de controle discretas. O tempo de processamento do caso 1 é de 25,8438 segundos, do caso 2 é de 19,9688 segundos e do caso 3 é de 476,047 segundos A Tabela 4 apresenta a comparação de indicadores do desempenho operacional da RD de 135 nós obtidos para os três estudos de caso considerados neste trabalho.

TABELA 4: INDICADORES OPERACIONAIS PARA A COMPARAÇÃo dos RESUlTAdOS ENTRE OS TRÊS ESTUdOS DE CASO Simulados PARA A RD DE 135 NÓS

\begin{tabular}{c|ccc}
\hline Indicador & Caso 1 & Caso 2 & Caso 3 \\
\hline$V_{\text {med. }}[\mathrm{p} . u]$. & 0,9999 & 1,0010 & 0,9999 \\
$V_{\text {min. }}[\mathrm{p} . \mathrm{u}]$. & 0,9833 & 0,9835 & 0,9818 \\
$V_{\text {max. }}[\mathrm{p} . \mathrm{u}]$. & 1,0164 & 1,0171 & 1,0142 \\
$P_{\text {med. }}^{\text {loss }}[\mathrm{kW}]$ & 378,99 & 226,27 & 326,24 \\
$P_{0, \text { med. }}[\mathrm{kW}]$ & $13.976,11$ & $13.823,40$ & $13.923,36$ \\
$Q_{0, \text { med. }}[\mathrm{kVAr}]$ & $4.949,90$ & $4.099,28$ & $1.962,52$ \\
\hline
\end{tabular}

A partir desses indicadores, pode-se perceber que o estudo de caso 3 resulta em valores médios para as magnitudes de tensão mais próximos de 1 p.u., com uma menor diferença entre as magnitudes de tensão máxima e mínima. Além disso, apesar de as perdas ativas médias na RD se aproximarem mais das do estudo de caso 1 do que das do estudo de caso 2, a demanda de potência reativa da RD no estudo de caso 3 é significativamente menor devido à injeção de potência reativa pelos inversores inteligentes, o que proporciona maiores margens de carregamento para o sistema elétrico de potência como um todo.

Os perfis das magnitudes de tensão nodal são apresentados para os três estudos de caso nas Figuras 8, 9 e 10.

As injeções de potência ativa e reativa por inversores inteligentes ao longo do horizonte de planejamento da RD para o estudo de caso 3 são apresentadas nas Figuras 12 e 13.

A convergência do algoritmo de tratamento das variáveis de controle discretas se deu em 15 iterações para o estudo de caso 3; o processo de convergência de algumas dessas variáveis para o estudo de caso 3 é apresentado na Tabela 5.

A Figura 11 apresenta os valores absolutos das funções objetivo da RD de 135 nós dos 3 casos estudados analisando o algoritmo de discretização da penalidade senoidal. A maior diferença entre os casos avaliados é o período das 17 horas do caso 1 que é de 0,0022. O algoritmo de discretização apresenta reposta semelhante nas RDs de 69 e 135

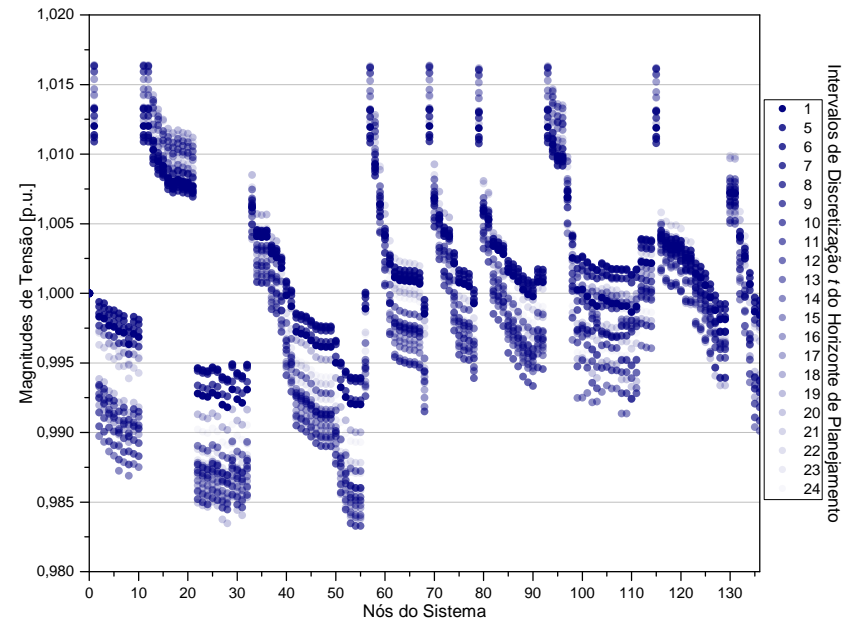

Figura 8: Magnitudes de tensão da RD de 135 nós para o estudo de caso 1 .

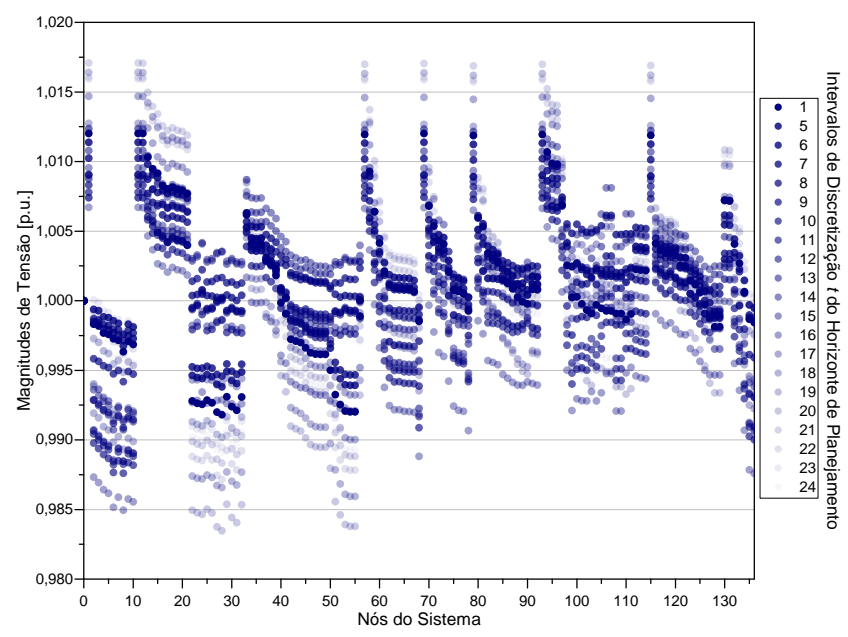

Figura 9: Magnitudes de tensão da RD de 135 nós para o estudo de caso 2 .

nós, mostrando a robustez da função penalidade senoidal na metodologia empregada.

Ressalta-se, por fim, que esta proposta consiste em um avanço das propostas apresentadas em $[10,11]$ dada a modelagem da injeção de potência ativa e reativa por inversores inteligentes que integram a GFD. A integração da GFD por inversores inteligentes viabiliza, pois, o aumento das opções de controle das RDs. Portanto, quaisquer comparações dos 
Tabela 5: Convergência do Algoritmo de Tratamento das Variáveis de Controle Discretas para a RD de 135 Nós no Estudo de CASO 3

\begin{tabular}{c|ccccccccccc}
\hline Iteração & $\gamma$ & $t_{0-1}^{(3)}$ & $t_{0-1}^{(10)}$ & $t_{0-1}^{(18)}$ & $t_{0-1}^{(20)}$ & $t_{0-1}^{(22)}$ & $b_{41}^{s h(1)}$ & $b_{69}^{s h(3)}$ & $b_{74}^{s h(16)}$ & $b_{111}^{s h(20)}$ & $b_{127}^{s h(22)}$ \\
\hline 0 & 0,0000100 & 0,958666 & 1,00554 & 0,942023 & 0,936234 & 0,940971 & 0,600000 & 0,286222 & 0,600000 & 0,352742 & 0,000000 \\
1 & 0,0000280 & 0,958676 & 1,00591 & 0,942575 & 0,936407 & 0,941149 & 0,600000 & 0,287594 & 0,600000 & 0,332966 & 0,000000 \\
2 & 0,0000784 & 0,958596 & 1,00613 & 0,942905 & 0,936506 & 0,941314 & 0,600000 & 0,287366 & 0,600000 & 0,328294 & 0,000515 \\
3 & 0,0002195 & 0,958275 & 1,00621 & 0,943167 & 0,936617 & 0,941885 & 0,600000 & 0,284356 & 0,600000 & 0,323222 & 0,013738 \\
4 & 0,0006147 & 0,957600 & 1,00623 & 0,943374 & 0,936754 & 0,942492 & 0,600000 & 0,281923 & 0,599989 & 0,318521 & 0,018320 \\
5 & 0,0017210 & 0,957018 & 1,00625 & 0,943573 & 0,936976 & 0,942964 & 0,600000 & 0,287557 & 0,600000 & 0,313457 & 0,013380 \\
6 & 0,0048189 & 0,956626 & 1,00625 & 0,943666 & 0,937212 & 0,943345 & 0,600000 & 0,293444 & 0,600000 & 0,307537 & 0,007446 \\
7 & 0,0134929 & 0,956408 & 1,00625 & 0,943715 & 0,937372 & 0,943576 & 0,600000 & 0,297132 & 0,600000 & 0,303350 & 0,003316 \\
8 & 0,0377802 & 0,956311 & 1,00625 & 0,943741 & 0,937450 & 0,943682 & 0,599999 & 0,298910 & 0,600000 & 0,301331 & 0,001300 \\
9 & 0,1057850 & 0,956272 & 1,00625 & 0,943747 & 0,937481 & 0,943725 & 0,599984 & 0,299594 & 0,600000 & 0,300499 & 0,000483 \\
10 & 0,2961970 & 0,956258 & 1,00625 & 0,943749 & 0,937493 & 0,943740 & 0,599993 & 0,299856 & 0,600000 & 0,300178 & 0,000172 \\
11 & 0,8293510 & 0,956253 & 1,00625 & 0,943750 & 0,937498 & 0,943747 & 0,599997 & 0,299949 & 0,600000 & 0,300064 & 0,000062 \\
12 & 2,3221800 & 0,956251 & 1,00625 & 0,943750 & 0,937499 & 0,943749 & 0,599999 & 0,299982 & 0,600000 & 0,300023 & 0,000022 \\
13 & 6,5021100 & 0,956250 & 1,00625 & 0,943750 & 0,937500 & 0,943750 & 0,600000 & 0,299993 & 0,600000 & 0,300008 & 0,000008 \\
14 & 18,205900 & 0,956250 & 1,00625 & 0,943750 & 0,937500 & 0,943750 & 0,600000 & 0,299998 & 0,600000 & 0,300003 & 0,000003 \\
15 & 50,976600 & 0,956250 & 1,00625 & 0,993750 & 0,937500 & 0,943750 & 0,600000 & 0,300000 & 0,600000 & 0,300000 & 0,000000 \\
\hline
\end{tabular}

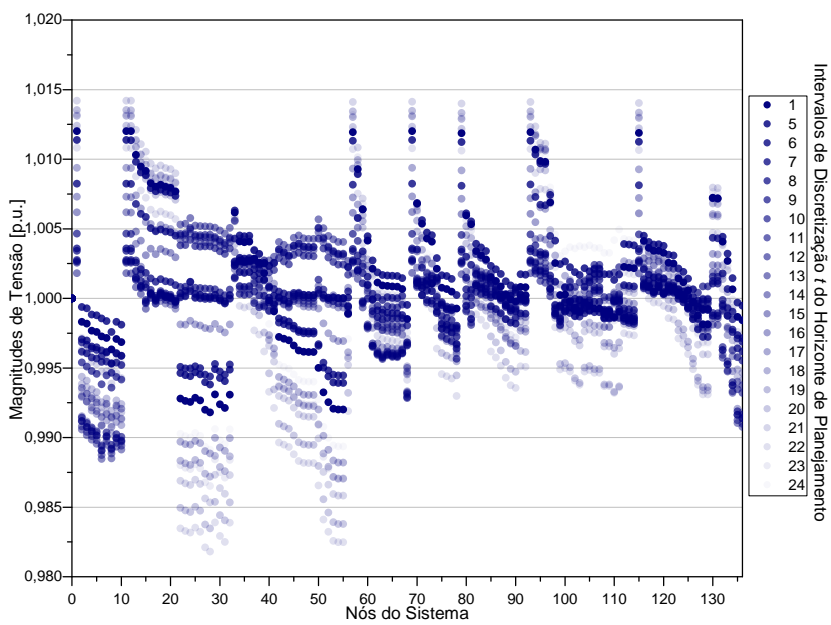

Figura 10: Magnitudes de tensão da RD de 135 nós para o estudo de caso 3 .

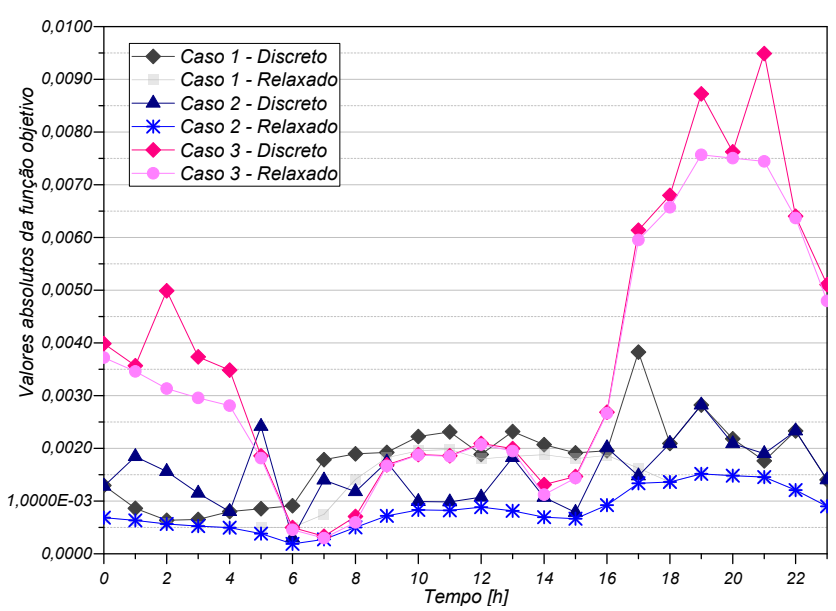

Figura 11: Valores da função objetivo para os casos avaliados do problema discreto e com relaxação contínua das variáveis de controle da RD de 135 nós.

resultados obtidos aos das propostas apresentadas em [10,11] são inviáveis.

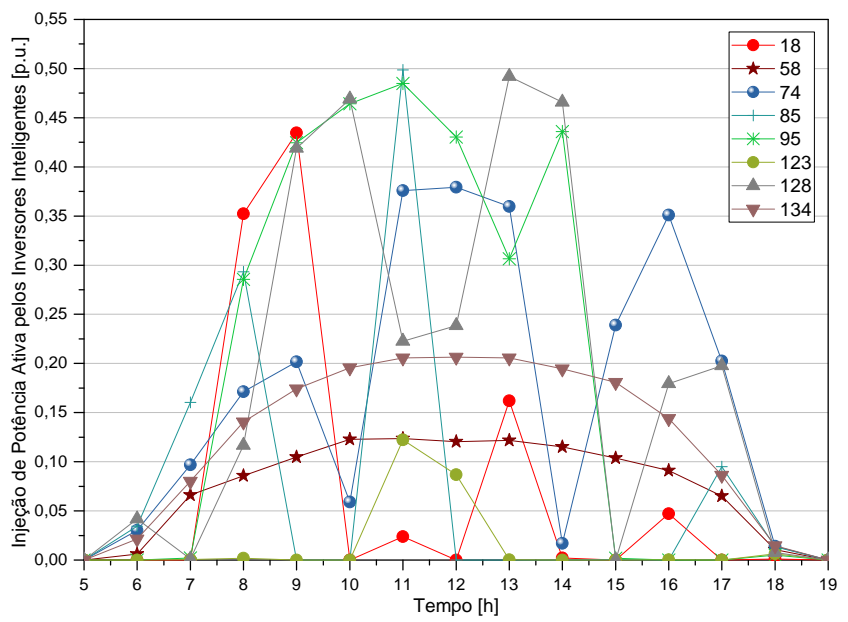

Figura 12: Perfis de injeção de potência ativa pelos inversores inteligentes da RD de 135 nós para o estudo de caso 3.

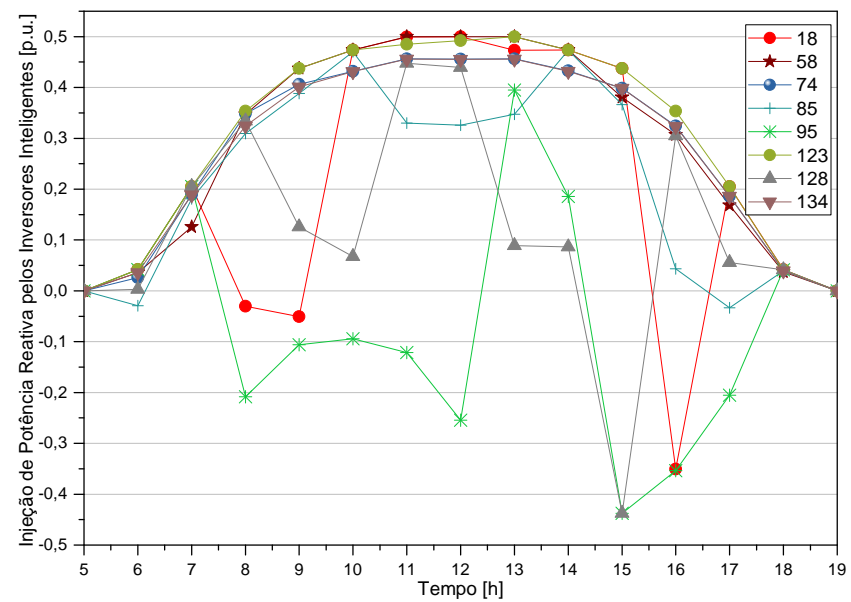

Figura 13: Perfis de injeção de potência reativa pelos inversores inteligentes da RD de 135 nós para o estudo de caso 3.

\section{Conclusões}

Este trabalho propôs um modelo para o problema de planejamento de véspera da operação horária de RDs com GFD integrada por inversores inteligentes por meio da formulação de um problema de OVV com variáveis de decisão con- 
tínuas e discretas. A metodologia empregada para a resolução deste problema de OVV consiste na modificação do problema original em um problema equivalente somente com variáveis de decisão contínuas e no tratamento das variáveis de decisão discretas por funções penalidade senoidal quadráticas. Pelos resultados obtidos, pôde-se concluir que a penetração da GFD integrada às RDs por inversores inteligentes diminui efetivamente os desvios de tensão nos alimentadores primários ao mesmo tempo que proporciona, de maneira geral, uma menor demanda de potência reativa pela $\mathrm{RD}$, o que resulta na ampliação das margens de carregamento do sistema elétrico como um todo.

Trabalhos futuros considerarão a limitação do número de ajustes nas variáveis de controle ao longo do horizonte de planejamento, a determinação de uma função objetivo que considere simultaneamente a minimização nos desvios de magnitude de tensão e a minimização das perdas na RD, e a característica estocástica da GFD para horizontes de planejamento mais longos.

\section{REFERENCIAS}

[1] H. Villarroel-Gutiérrez, "The Argentine electrical sector and its trends toward renewable resources," IEEE Latin America Transactions, vol. 17, no. 10, pp. 1625-1636, 2019.

[2] P. Benalcázar, J. Lara, and M. Samper, "Distributed photovoltaic generation in Ecuador: economic analysis and incentives mechanisms," IEEE Latin America Transactions, vol. 18, no. 3, pp. 564-572, 2020.

[3] F. Katiraei and J. R. Aguero, "Studies for utility-scale photovoltaic distributed generation," IEEE Power and Energy Magazine, vol. 9, no. 3, pp. 62-71, 2011.

[4] V. Vita, T. Alimardan, and L. Ekonomou, "The impact of distributed generation in the distribution networks' voltage profile and energy losses," in 2015 IEEE European Modelling Symposium (EMS), 2015 pp. 260-265.

[5] J. Hou, Y. Xu, J. Liu, L. Xin, and W. Wei, "A multi-objective volt-var control strategy for distribution networks with high PV penetration," in 10th International Conference on Advances in Power System Control, Operation \& Management (APSCOM 2015), 2015, pp. 1-6.

[6] I. Lepadat, E. Helerea, S. Abagiu, and C. Mihai, "Impact of distributed generation on voltage profile and power losses in a test power grid," in 2017 International Conference on Optimization of Electrical and Electronic Equipment (OPTIM) \& 2017 International Aegean Conference on Electrical Machines and Power Electronics (ACEMP), 2017, pp. 128-133.

[7] G. Gutiérrez, A. Aguila, D. González, and L. Ortiz, "Optimum location and sizing of capacitor banks using VOLT VAR compensation in micro-grids," IEEE Latin America Transactions, vol. 18, no. 3, pp $465-472,2020$

[8] B. Seal, Common Functions for Smart Inverters, 4th ed. Electric Power Research Institute, 2016.

[9] A. Molina-García, R. A. Mastromauro, T. García-Sánchez, S. Pugliese, M. Liserre, and S. Stasi, "Reactive power flow control for PV inverters voltage support in LV distribution networks," IEEE Transactions on Smart Grid, vol. 8, no. 1, 2017.

[10] F. M. de Vasconcelos, G. R. M. da Costa, and G. G. Lage, "An NLP penalty-based strategy for handling discrete controls for Volt/Var optimization in distribution systems," in 2015 IEEE Power \& Energy Society General Meeting, 2015, pp. 1-5.

[11] M. B. Liu, C. A. Cañizares, and W. Huang, "Reactive power and voltage control in distribution systems with limited switching operations," IEEE Transactions on Power Systems, vol. 24, no. 2, pp. 889-899, 2009

[12] P. Belotti, C. Kirches, S. Leyffer, J. Linderoth, J. Luedtke, and A. Mahajan, "Mixed-integer nonlinear optimization," Acta Numerica, vol. 22, pp. 1-131, 2013.
[13] E. M. Soler, V. A. de Sousa, and G. R. M. da Costa, "A modified primal-dual logarithmic-barrier method for solving the optimal power flow problem with discrete and continuous control variables," European Journal of Operational Research, vol. 222, no. 3, pp. 616-622, 2012.

[14] E. M. Soler, E. N. Asada, and G. R. M. da Costa, "Penalty-based nonlinear solver for optimal reactive power dispatch with discrete controls," IEEE Transactions on Power Systems, vol. 28, no. 3, pp. 2174 $2182,2013$.

[15] L. Liu, X. Wang, X. Ding, and H. Chen, "A robust approach to optimal power flow with discrete variables," IEEE Transactions on Power Systems, vol. 24, no. 3, pp. 1182-1190, 2009.

[16] Mingbo Liu, S. K. Tso, and Ying Cheng, "An extended nonlinear primal-dual interior-point algorithm for reactive-power optimization of large-scale power systems with discrete control variables," IEEE Transactions on Power Systems, vol. 17, no. 4, pp. 982-991, 2002.

[17] F. Capitanescu and L. Wehenkel, "Sensitivity-based approaches for handling discrete variables in optimal power flow computations," IEEE Transactions on Power Systems, vol. 25, no. 4, pp. 1780-1789, 2010 .

[18] R.-H. Liang, Y.-K. Chen, and Y.-T. Chen, "Volt/Var control in a distribution system by a fuzzy optimization approach," International Journal of Electrical Power \& Energy Systems, vol. 33, no. 2, pp. 278-287, 2011

[19] Z. Hu, X. Wang, H. Chen, and G. A. Taylor, "Damping subsynchronous resonance," Engineering and Technology, vol. 9, no. 4, pp. $431-442,2002$.

[20] F. B. Prioste and G. R. Alvares, "Impactos da interligação de uma usina solar fotovoltaica com controle ativo de tensão em um sistema de distribuição," in XIV Simpósio de Especialistas em Operação e Planejamento da Expansão Elétrica (SEPOPE), 2018, pp. 1-11.

[21] Q. Long, J. Wang, D. Lubkeman, N. Lu, and P. Chen, "Volt-Var optimization of distribution systems for coordinating utility voltage control with smart inverters," in 2019 IEEE Power \& Energy Society Innovative Smart Grid Technologies Conference (ISGT), 2019, pp. 1-5.

[22] C. Li, V. R. Disfani, H. V. Haghi, and J. Kleissl, "Coordination of oltc and smart inverters for optimal voltage regulation of unbalanced distribution networks," Electric Power Systems Research, vol. 187, p. 106498, 2020. [Online]. Available: http://www.sciencedirect.com/ science/article/pii/S0378779620303011

[23] E. R. S. de Ferreira, R. M. Barros, T. A. R. d. Silva, R. A. L. de Rabêlo, V. R. Júnior, and G. G. Lage, "Application of a data communication infrastructure for the voltage magnitude control in transmission power systems," in 2019 IEEE International Conference on Systems, Man and Cybernetics (SMC), 2019, pp. 4308-4315.

[24] V. Loia, A. Vaccaro, and K. Vaisakh, "A self-organizing architecture based on cooperative fuzzy agents for smart grid voltage control,' IEEE Transactions on Industrial Informatics, vol. 9, no. 3, pp. 1415$1422,2013$.

[25] M. Ibrahim and M. M. A. Salama, "Smart distribution system volt/var control using distributed intelligence and wireless communication," IET Generation, Transmission Distribution, vol. 9, no. 4, pp. 307-318, 2015 .

[26] S. S. Rangarajan, E. R. Collins, and J. C. Fox, "Efficacy of a smart photovoltaic inverter as a virtual detuner for mitigating network harmonic resonance in distribution systems," Electric Power Systems Research, vol. 171, pp. 175 - 184, 2019. [Online]. Available: http: //www.sciencedirect.com/science/article/pii/S0378779619300562

[27] M. E. Baran and F. F. Wu, "Optimal capacitor placement on radial distribution systems," IEEE Transactions on Power Delivery, vol. 4, no. 1 , pp. $725-734,1989$.

[28] R. Kavasseri and C. Ababei, "REDS: REpository of Distribution Systems," http://www.dejazzer.com/reds.html, 2020.

[29] E. N. M. Silva, A. B. Rodrigues, and M. da Guia da Silva, "Stochastic assessment of the impact of photovoltaic distributed generation on the power quality indices of distribution networks," Electric Power Systems Research, vol. 135, pp. 59-67, 2016.

[30] AMPL, Optimization Inc., “AMPL,” http://www.ampl.com, 2017. 
[31] R. Fourer, D. M. Gay, and B. W. Kernighan, $A M P L$, 2nd ed. Duxbury Press, 2002.

[32] ARKI Consulting \& Development A/S, "CONOPT," http://www.conopt.com, 2019. 